\title{
Evaluating performance of metagenomic characterization algorithms using in silico datasets generated with FASTQSim
}

\author{
Anna Shcherbina ${ }^{1}$, Darrell O. Ricke ${ }^{* 1}$, Nelson Chiu $^{1}$ \\ *Corresponding author: Darrell Ricke \\ ${ }^{1}$ MIT Lincoln Laboratory \\ Bioengineering systems and Technologies Group \\ 244 Wood St \\ Lexington, MA, 02420 \\ Author e-mail addresses: \\ - Anna Shcherbina - annashch@stanford.edu \\ - Darrell Ricke - Darrell.Ricke@ll.mit.edu \\ - Nelson Chiu - Nelson.Chiu@II.mit.edu
}

This work is sponsored by the Assistant Secretary of Defense for Research and Engineering under Air Force Contract \#FA8721-05-C-0002. Opinions, interpretations, recommendations and conclusions are those of the authors and are not necessarily endorsed by the United States Government. 


\section{Abstract}

\section{Background}

In silico bacterial, viral, and human truth datasets were generated to evaluate available metagenomics algorithms. Sequenced datasets include background organisms, creating ambiguity in the true source organism for each read. Bacterial and viral datasets were created with even and staggered coverage to evaluate organism identification, read mapping, and gene identification capabilities of available algorithms. These truth datasets are provided as a resource for the development and refinement of metagenomic algorithms. Algorithm performance on these truth datasets can inform decision makers on strengths and weaknesses of available algorithms and how the results may be best leveraged for bacterial and viral organism identification and characterization.

Source organisms were selected to mirror communities described in the Human Microbiome Project as well as the emerging pathogens listed by the National Institute of Allergy and Infectious Diseases. The six in silico datasets were used to evaluate the performance of six leading metagenomics algorithms: MetaScope, Kraken, LMAT, MetaPhIAn, MetaCV, and MetaPhyler.

\section{Results}

Algorithms were evaluated on runtime, true positive organisms identified to the genus and species levels, false positive organisms identified to genus and species level, read mapping, relative abundance estimation, and gene calling. No algorithm out performed the others in all categories, and the algorithm or algorithms of choice strongly depends on analysis goals. MetaPhIAn excels for bacteria and LMAT for viruses. The algorithms were ranked by overall performance using a normalized weighted sum of the above metrics, and MetaScope emerged as the overall winner, followed by Kraken and LMAT.

\section{Conclusions}

Simulated FASTQ datasets with well-characterized truth data about microbial community composition reveal numerous insights about the relative strengths and weaknesses of the metagenomics algorithms evaluated. The simulated datasets are available to download from the Sequence Read Archive (SRP062063).

\section{Keywords}

FASTQsim, Metagenomics, in silico, evaluation, Kraken, LMAT, MetaPhIAn, MetaPhyler, MetaScope, MetaCV

\section{Background}

Continuing advances in sequencing technologies are increasing the feasibility of sequencing entire microbial communities rather than individual organisms. This has led to rapid developments in the field of metagenomics aimed at studying genomic material recovered directly from environmental and medical samples. Sequencing the metagenome enables the capture of greater genetic diversity than can be sampled with highly targeted approaches such as microarrays. Metagenomic sequencing has a number of applications for medical diagnostics (i.e. human gut microbiome analysis), environmental profiling (i.e. soil samples), and homeland defense[1-3]. Metagenomic techniques also enable the study of communities of organisms simulated in vitro[4]. 
sample data. They employ a variety of techniques to achieve the opposing goals of high accuracy and low runtime. In this study, the performance of these varied approaches to metagenomic sequence classification was evaluated on a suite of in silico datasets with perfectly characterized composition. MetaScope, winner of the Defense Threat Reduction Agency's Grand Challenge[5], relies on sequence analysis using spaced seeds followed by an augmented least common ancestor algorithm to map reads and assign genes for input FASTQ samples[6, 7]. Kraken[8] uses exact alignment of k-mers in combination with an optimized database and another version of the least common ancestors algorithm. MetaPhIAn[9] relies on unique clade-specific marker genes identified from 3000 reference genomes. The Livermore Metagenomic Analysis Toolkit (LMAT) exploits genetic relationships between different organisms by pre-computing the occurrence of each short sequence across the entire reference database and storing the evolutionarily conserved sequence patterns[10-12]. MetaCV translates nucleotide sequences into six frame peptides, which are then decomposed into k-mers. The k-mer frequency is computed in a protein-reference database and used to assign k-mer weights[13]. Finally, MetaPhyler uses a precomputed database of reference phylogenetic marker genes to build a sequence classifier. The classifier, based on BLAST, uses trained thresholds for various combinations of taxonomic ranks, sequence length, and reference genomes[14].

Simulated in silico datasets are a valuable tool for metagenomic research and provide capabilities to evaluate algorithm performance as well as to test hypotheses that cannot be examined through empirical observation. For example, simulated data has revealed biases and heterogeneity in the estimation of diversity metrics from metagenomics samples[15]. Additionally, multiple studies have demonstrated the usefulness of simulated metagenomics datasets for benchmarking sequence assembly and gene prediction pipelines[16-18]. Simulated datasets are also an effective means of parameter optimization for improved algorithm performance and can be used to optimize study design. Sequence simulation can aid with answering questions about coverage requirements, necessary sequence length, and whether paired-end or single-end sequencing should be used. For example, the ART simulator was successfully used by the 1000 Genomes Project Consortium to examine the effects of read length and PE insert size on a read's ability to map to the human genome[19].

In this study, six in silico datasets were simulated by the FASTQsim tool. Figure S1 illustrates the composition of each dataset. These datasets contained sequences from reference bacterial and viral genomes, as most human pathogens are members of these taxa. The HMP Even and HMP Staggered datasets were generated to include sequences from the 20 organisms from the Human Microbiome Project[20] (Supplementary Table 1). The HMP organisms were selected for inclusion after an attempt to benchmark the performance of MetaScope with the HMP dataset revealed potential contamination in the dataset. As the HMP benchmark dataset was generated by sequencing organisms cultured in vitro, there was no absolute truth for any background contaminant organisms in the dataset and it was not possible to determine whether the contamination was real or whether MetaScope was calling false positive organisms.

The bacterial dataset (Supplementary Table 2) was designed to test algorithm specificity. Four genera of pathogens were selected from the National Institute of Allergy and Infectious Diseases list of biodefense and emerging infectious disease agents[21] due to their relevance to disease diagnostics from metagenomics samples. These included Yersinia, Coxiella, Brucella, and Salmonella. Additionally, the Escherichia genus was added to the list due to the high abundance of representative sequences in GenBank[22].

Two virus datasets were generated with 21 species across 11 representative genera (Supplementary 
Table 3). As with the bacterial dataset, candidates were selected due to their inclusion on the NIAID list of emerging pathogens (Marburg virus, Machupo virus, Sudan ebolavirus, Junin virus, Guanarito virus, Chapare virus, Omsk hemorrhagic fever virus) as well as abundance of representative organisms in GenBank (HIV 1, HIV 2, Influenza A virus).

Finally, a dataset of human reads from build GRCh38 at 10x (22 million reads) coverage was generated to test host-filtering capabilities of each algorithm. This dataset was generated to measure how well algorithms can overcome the challenges posed by human sequence contamination in public reference databases[23]. For example, endogenous retroviral remnants may be incorrectly classified as belonging to viral genomes in a sample[24-26].

\section{Methods}

\section{Improvements to FASTQsim}

The FASTQsim toolkit was augmented to annotate gene information for simulated reads[27]. The "FASTQmapGenes" functionality was added, allowing users to specify NCBI accession ids to use for annotating gene information in simulated reads. The FASTQsim toolkit uses the Entrez and SeqIO libraries from BioPython[28] to download the specified files from GenBank in .gb format. The GenbankParser[29] java application is then used to parse the .gb files in order to extract all information encoded in the CDS and Gene tags. These gene and CDS annotations are appended to the headers within the simulated FASTQ files generated by FASTQsim, such that all reads that fall within a CDS or gene region are annotated with the corresponding CDS and gene information.

\section{In silico data generation}

The FASTQsim toolkit was used to generate six in silico datasets. All were generated with the Illumina error and read length profile included with FASTQsim version 2.0, with no host background added. Specifically, read length of 150 bases was used, with single base mutation, insertion, and deletion rates as specified in the FASTQsim v. 2.0 documentation (http://sourceforge.net/p/fastqsim/code/ci/master/tree/params/illumina/). NCBI identifiers for all input data are listed in Supplementary Tables 1-3. The Krona toolkit[30] was used to visualize evaluation dataset composition.

Two in silico datasets were generated - "HMP Even" and "HMP Staggered" (Supplementary Table 1). For the HMP even dataset, FASTQsim was executed to provide equal number of reads for each species of organism (approximately 60,000 reads per species), with one exception -- 559 reads for Streptococcus agalactiae were added to simulate a low-level contaminant organism. Version 2.0 of the FASTQsim algorithm probabilistically simulated read counts and error distributions based on a provided model. Due to the probabilistic nature of the algorithm, coverage levels deviated slightly from the specified 60,000 reads, with the largest deviation observed for the E. faecalis organism (52,290 reads). For the HMP Staggered dataset, coverage levels varied from $11.3 \times$ (217,512 reads) for Actinomyces odontolyticus to $0.001 \times$ ( 2 reads) for Neisseria meningitidis. The goal of the staggered dataset was to evaluate the ability of metagenomic algorithms to detect organisms present at very low concentrations, i.e. less than 5 reads.

The bacterial dataset included reads from the genear Yersinia, Coxiella, Brucella, Salmonella, and Escherichia. For each of the five genera, several representative species were selected (i.e., Brucella abortus, Brucella melitensis, Brucella suis). Next, several representative strains were selected for each species (i.e. Brucella melitensis ATCC 23457, Brucella melitensis biovar abortus 2308, Brucella melitensis 
biovar 1 strain 16M, and Brucella melitensis M28). Organisms were spiked into a FASTQ dataset with coverage levels ranging from $10 x$ to $0.00002 x$ ( 1 read).

For the Virus Even dataset, 10x coverage of each organism was simulated. For the Virus Staggered dataset, coverage varied from $100 x$ for Sudan ebolavirus to $0.5 x$ for the Human coronavirus HKU1.

\section{Metagenomic algorithm execution}

Six metagenomic algorithms were selected for execution on the evaluation datasets. These included:

- MetaScope - winner of the Defense Threat Reduction Agency's Grand Challenge[7] (version 2.0)

- MetaPhIAn[9] (version 1.7.8, https://bitbucket.org/nsegata/MetaPhIAn/src/),

- MetaCV[13] (version 2.3.0, http://sourceforge.net/projects/metacv/files/),

- MetaPhyler[14] (version 1.13, http://MetaPhyler.cbcb.umd.edu/\#download),

- Kraken[8] (v0.10.5, https://ccb.jhu.edu/software/kraken/),

- $\operatorname{LMAT}[10-12]$ (v1.2.5, http://sourceforge.net/projects/lmat/).

All algorithms were executed on each of the evaluation datasets using a machine with $512 \mathrm{~GB}$ of RAM, 64 cores, 1 TB hard drive, running the Fedora 17 operating system. All algorithms were executed with the default set of databases described in their respective documentation, downloaded on March 1, 2015. Algorithms were evaluated using 60 of the 64 available cores.

Attempts were also made to install and run the SURPI (v1.0, https://github.com/chiulab/surpi)[31] and compressed BLAST (v0.9, http://cast.csail.mit.edu/)[32] algorithms, but these were unsuccessful.

\section{Algorithm performance evaluation}

Runtime in seconds, true positive genus and species calls, false positive genus and species calls, read mapping, and relative abundance results at the species level were computed for all algorithm results. Additionally, correct gene calls were calculated for the set of algorithms that provided gene calling results (MetaScope, MetaCV, LMAT). The Gene ID Conversion function in the DAVID Bioinformatics Database[33] was used to convert across gene representation formats utilized by the three algorithms. Genes were marked as true positives if they matched the gene id, official gene symbol, locus tag, protein id, or specific product name of the truth data.

\section{Availability of supporting data}

The FASTQsim toolkit can be downloaded from SourceForge: http://sourceforge.net/projects/fastqsim/

In silico evaluation datasets can be downloaded from the Sequence Read Archive: SRP062063

SRR2146185 - Virus Staggered dataset

SRR2146184-- Virus Even dataset

SRR2146183-Bacterial dataset

SRR2146181-HMP Staggered dataset

SRR2146182 - HMP Even dataset

\section{Results and Discussion}

Runtime in seconds, true positive genus and species identification, false positive genus and species identification, and false negative species calls were determined for each of the metagenomic algorithms (Figure 1). Among the algorithms evaluated, only MetaScope mapped a small number of reads in our datasets to a taxon rank below species. Consequently, although the initial focus of the Bacterial dataset 
was to assess the ability of the algorithms to distinguish between different strains of the same species, it was decided to evaluate both true and false positives at species and genus level. To determine an overall rank of the algorithms across the datasets, the area occupied by each in the radar plot was computed (Table 1). When the polygon area was calculated using the MATLAB polyarea function and summed across all datasets, MetaScope emerges as the winner, with the largest overall area. Kraken and LMAT are the runner-ups, and MetaPhyler performed the worst. In addition to the algorithms' rank overall, several trends can be noted in the individual performance categories. the shortest runtime. The algorithm had the fastest time on the three bacterial datasets $-22.64 \mathrm{~s}$ for HMP Even, $53.3 \mathrm{~s}$ on HMP staggered, and $220 \mathrm{~s}$. on Bacteria. The second fastest times for these three datasets were 5 to 10 times slower: $233 \mathrm{~s}$ (MetaPhIAn), $261 \mathrm{~s}$ (MetaScope), and 2,700 s (LMAT), respectively. MetaPhIAn is able to execute quickly partly because it does not perform a host-filtering step. MetaPhIAn came in second for the virus datasets, with a runtime of 11 seconds on both, compared to 9 and 7 seconds for Kraken. MetaPhIAn failed to run on the human dataset. Kraken, MetaScope, and LMAT exhibited similar runtimes on all datasets, averaging $353 \mathrm{~s}$ on HMP Even, $354 \mathrm{~s}$ on HMP staggered, and 3,595 s on Bacteria. On the other end of the spectrum, MetaPhyler was an outlier for high runtime, requiring 15,480 s on HMP Even, 19,231 s on HMP staggered, and 129,600 s on Bacteria.

In addition to its high speed, MetaPhIAn also achieved the highest accuracy, defined as ratio of true positives to false positives, on the bacterial datasets. It identified all 20 species in the HMP even dataset with only a single false positive organism. On HMP staggered, it missed 4 species out of 20 but reported only 2 false positive species. MetaScope, the runner up, reported a single false negative species but 414 false positives. However, the MetaPhIAn reference database is customized for bacteria, and no support exists at the time of this writing for profiling viruses or eukaryotes. MetaScope achieved the second- highest ratio of true positives to false positives, reporting slightly more true positives and approximately half as many false positives as Kraken. LMAT was the least conservative and reported the highest number of false positive organisms. MetaPhyler made highly conservative calls-false positives were low, but so were true positives. Additionally, MetaPhyler, and MetaCV, as well as MetaPhIAn, did not report results for the viral datasets.

Algorithm performance on the Human dataset (Figure 2k) illustrates the efficacy of the host-filtering step for each algorithm. The human reference genome is incomplete[34, 35] and misses regions specific to individual host subjects. These missed regions show up as false positives on the Human evaluation dataset - algorithms assign them to organisms other than the human host because these reads are not removed during the host filtering step. For example, MetaScope reports 152 organisms, with fewer than 100 reads assigned to each. Kraken has a similar false positive profile; it reports 1,266 species that account for $<1 \%$ of the reads in the dataset. MetaCV reports 2,998 false organisms with low read count, and LMAT reports 1,118 species that account for less than $0.01 \%$ of the reads. MetaPhyler does not report results more specific than the Class taxonomy level for the Human dataset, in line with the conservative approach of this algorithm. MetaPhIAn crashes with a segmentation fault on the Human dataset, which most likely is an artifact of the non-host-filtering approach used by this algorithm. 
classified the highest number of reads correctly for both the genus and species level, and cluster closest to the truth in the dendrogram. However, for the viral datasets, LMAT performed best, classifying the most reads correctly.

Although the Actinomyces odontolyticus (NZ_DS264586.1) organism had the highest coverage (11.3x, 217512 reads) in the HMP staggered dataset, the algorithms on the whole did not perform well on this organism. It was not identified by the Kraken, MetaCV, and MetaPhyler algorithms, and called at a low level by MetaScope (153 reads) (Figure 2g) MetaCV mapped the most reads correctly -108,211 (49.7\%) and MetaPhIAn was second best, identifying 22,647 (10.4\%) of the reads. None of the algorithms identified any of the 2,045 A. odontolyticus genes (Figure $\mathbf{5 b}$ ). This poor performance likely results from the fact that $A$. odontolyticus genome annotation in GenBank is incomplete[36]. Conversely, at the species level, five of the six algorithms mapped a high number of reads to Streptococcus agalactiae for both the HMP even and HMP staggered datasets (Figure $\mathbf{2 f}, \mathbf{2 g}$ ), but only a small number of reads for this organism were present in the truth data. The relative abundance of Streptococcus mutans is lower in the algorithm calls as compared to truth, while the relative abundance of Streptococcus agalactiae is higher, suggesting that a number of the reads called for $S$. agalactiae are actually from S. mutans (Figure 3b, 3d). This implies difficulty distinguishing between closely related species. Similarly, a high number of reads are assigned correctly to the Yersinia and Escherichia genera by Kraken and MetaScope (Figure 2c.) However, the algorithms under-assign reads for Escherichia albertii and overassign reads for Yersinia pseudotuberculosis, which indicates difficulty in distinguishing between these species (Figure $2 \mathrm{~h}$ ).

Overall, algorithms were equally as able to identify organisms in the staggered datasets as in the even datasets, suggesting that accurate read mapping depends more on the database supplied to the algorithm rather than the abundance of the organism in the dataset. Additionally, for the bacterial datasets, Kraken, MetaScope, LMAT, and MetaPhIAn generally agreed on read mapping assignments. However, for the viral datasets, the algorithms missed different sets of organisms - i.e., in Figure $\mathbf{3 i}$, LMAT failed to map reads for HIV1, Influenza A virus, Marburg virus, and Machupo virus, whereas MetaScope and Kraken correctly mapped reads for these organisms. However, MetaScope and Kraken both failed to map reads for Human papillomavirus 5, SARS coronavirus, Human papillomavirus 32, and Canine papillomavirus 3, while LMAT succeeded in mapping reads for these organisms. This suggests that for viral datasets, it might be worthwhile to execute both LMAT and one of Kraken or MetaScope, and calculate the union of the results.

The algorithms were also evaluated based on false positive hits (Figure 4). MetaCV and LMAT have diverse error profiles - small numbers of reads are mapped to a high number of false positive organisms. Our past experiences with the MetaScope algorithm suggest that this false positive profile indicates an algorithm has difficulty classifying organisms that are not present in the reference database. Ideally, when an algorithm encounters a novel organism, it should regress up the taxonomic tree until a nearest neighbor for the unknown organism can be established. However, the algorithm may instead report all reference organisms that match the unknown sample to a certain threshold. In contrast, Kraken has a highly concentrated error profiles; fewer than 20 false positive organisms are reported, but several thousand reads are mapped to each of them, suggesting high confidence calls. Figure $\mathbf{4 c}$ and $\mathbf{4 d}$ summarizes the top 20 organisms in terms number of mapped reads, indicating high agreement b e $\mathrm{t}$ w e e $\mathrm{n}$ Kraken and MetaScope. On the list of false positive genera are several members of the Enterobacteriaceae family, including Shigella, Klebsiella, and Enterobacter. The true positive genera Salmonella, Escherichia, and Yersinia are members of this family as well. More difficult to explain is the 
presence of the Methanolobus genus, which is a member of the kingdom Archaea and is distantly related to the bacteria in the truth data.

For the viral datasets, MetaCV returned a high number of false positives and exhibited poor performance. Kyasanur forest disease virus, a close relative to the true positive Omsk hemorrhagic fever virus was the sole false positive for LMAT, and MetaScope did not report any false positive organisms for either viral dataset.

Finally, the gene calling capabilities of the algorithms were evaluated (Figure 5). Only MetaScope, LMAT, and MetaCV call genes, so these three were included for analysis. For the HMP Even/Staggered, Bacteria, and Virus Staggered datasets, MetaScope identified the most genes correctly out of the three algorithms. LMAT identified more correct genes on the Virus Even dataset (101, compared to 93 for MetaScope).

\section{Conclusions}

In summary, in silico datasets with known truth data for read and gene distribution across different taxons serve as a valuable tool for evaluating algorithm performance. The HMP Even/Staggered, Bacteria, Virus Even/Staggered, and Human datasets generated with FASTQsim elucidate multiple patterns in performance for leading metagenomics algorithms. No algorithm out performed the others in all categories, and the algorithm of choice strongly depends on analysis goals. For bacterial datasets, MetaPhIAn is a clear winner, achieving the lowest runtime, highest ratio of true positives to false positives, and the most precise read mapping. However, MetaPhIAn does not assign genes and does not work on taxons other than Bacteria. LMAT is a clear winner for viral datasets in terms of accuracy, and also provides gene calling functionality. The algorithm most closely matched the relative abundance profile of the truth genera and species across all datasets. However, LMAT also reported the highest rate of false positive genera and species calls on the bacterial datasets. Kraken and MetaScope were the runners up in terms of runtime, ratio of true positives to false positives, and read mapping. MetaScope also performed best for gene mapping, which Kraken does not do. These algorithms performed solidly across all categories evaluated and can be applied most universally across versatile metagenomic applications. MetaPhyler and MetaCV came in last for runtime, ratio of true positives to false positives, and read mapping. They also do not provide results out of the box for viral datasets.

Although viral, bacterial, and human datasets were simulated for this study, the techniques described here can be extended to evaluate metagenomic algorithm performance for other taxa. For example, fungal contamination incidents at medical facilities such as the 2012 incident at the New England Compounding Center[37] can be contained more quickly and effectively with the aid of metagenomic sequencing. Other potential applications include rapid diagnosis of parasite infections[38]. 


\section{List of abbreviations}

GB - gigabyte

RAM - random-access memory

$s-$ seconds

TB - terabyte

$x-$ fold coverage

\section{Competing interests}

The authors declare that they have no competing interests.

\section{Ethics Committee Approval}

Ethics approval was not required for this study because all data was generated in silico using references available in GenBank, as indicated in Supplementary Tables 1-3.

\section{Authors' contributions}

AS implemented FASTQSim updates and generated in silico datasets. AS and NC benchmarked algorithm performance on evaluation datasets. AS and DR wrote the manuscript. DR conceived of the study. All authors read and approved the final manuscript.

\section{References}

[1] H. G. Martin, N. Ivanova, V. Kunin, F. Warnecke, K. W. Barry, A. C. McHardy, et al., "Metagenomic analysis of two enhanced biological phosphorus removal (EBPR) sludge communities," Nat Biotech, vol. 24, pp. 1263-1269, 10//print 2006.

[2] G. W. Tyson, J. Chapman, P. Hugenholtz, E. E. Allen, R. J. Ram, P. M. Richardson, et al., "Community structure and metabolism through reconstruction of microbial genomes from the environment," Nature, vol. 428, pp. 37-43, 03/04/print 2004.

[3] J. C. Venter, K. Remington, J. F. Heidelberg, A. L. Halpern, D. Rusch, J. A. Eisen, et al., "Environmental Genome Shotgun Sequencing of the Sargasso Sea," Science, vol. 304, pp. 66-74, 2004.

[4] J. L. Morgan, A. E. Darling, and J. A. Eisen, "Metagenomic Sequencing of an In Vitro-Simulated Microbial Community," PLoS ONE, vol. 5, p. e10209, 2010.

[5] InnoCentive. (2013). Identifying organisms from a stream of DNA sequences. Available: https://www.innocentive.com/ar/challenge/9933138

[6] L. Ilie and S. Ilie, "Multiple spaced seeds for homology search," Bioinformatics, vol. 23, pp. 29692977, 2007.

[7] B. Buchfink, C. Xie, and D. H. Huson, "MetaScope - Fast and accurate identification of microbes in metagenomic sequencing data.," arXiv.org, submitted.

[8] D. E. Wood and S. L. Salzberg, "Kraken: ultrafast metagenomic sequence classification using exact alignments," Genome Biology, vol. 15, 2014.

[9] N. Segata, L. Waldron, A. Ballarini, V. Narasimhan, O. Jousson, and C. Huttenhower, "Metagenomic microbial community profiling using unique clade-specific marker genes," Nat Meth, vol. 9, pp. 811-814, 08//print 2012.

[10] S. Ames, J. E. Allen, D. A. Hysom, G. S. Lloyd, and M. B. Gokhale, "Design and Optimization of a Metagenomics Analysis Workflow for NVRAM," in Parallel \& Distributed Processing Symposium Workshops (IPDPSW), 2014 IEEE International, 2014, pp. 556-565.

[11] S. K. Ames, D. A. Hysom, S. N. Gardner, G. S. Lloyd, M. B. Gokhale, and J. E. Allen, "Scalable metagenomic taxonomy classification using a reference genome database," Bioinformatics, vol. 29, pp. 2253-2260, 2013. 
[12] B. Van Essen, H. Hsieh, S. Ames, and M. Gokhale, "DI-MMAP: A High Performance Memory-Map Runtime for Data-Intensive Applications," in High Performance Computing, Networking, Storage and Analysis (SCC), 2012 SC Companion:, 2012, pp. 731-735.

[13] J. Liu, H. Wang, H. Yang, Y. Zhang, J. Wang, F. Zhao, et al., "Composition-based classification of short metagenomic sequences elucidates the landscapes of taxonomic and functional enrichment of microorganisms," Nucleic Acids Research, vol. 41, 2013.

[14] B. Liu, T. Gibbons, M. Ghodsi, T. Trengen, and M. Pop, "Accurate and fast estimation of taxonomic profiles from metagenomic shotgun sequences," BMC Genomics, vol. 12, 2010.

[15] G. Bonilla-Rosso, "Lessons learned from simulated metagenomic datasets," Encyclopedia of Metagenomics, pp. 1-8, 2014.

[16] K. Mavromatis, N. Ivanova, K. Barry, H. Shapiro, E. Goltsman, A. McHardy, et al., "Use of simulated data sets to evaluate the fidelity of metagenomic processing methods," Nature Methods, vol. 4, pp. 495-500, 2007.

[17] M. Pignatelli and A. Moya, "Evaluating the fidelity of de novo short read metagenomic assembly using simulated data," PLOS One, 2011.

[18] D. Mende, A. Waller, S. Sunagawa, A. Jarvelin, M. Chan, M. Arumugam, et al., "Assessment of metagenomicm assembly using simulated next generation sequencing data," PLOS one, 2012.

[19] W. Huang, L. Li, J. Myers, and G. Marth, "ART: a next-generation sequencing read simulator," Bioinformatics, vol. 28, pp. 593-594, 2011.

[20] The NIH HMP Working Group, "The NIH Human Microbiome Project," Genome Research, vol. 19, pp. 2317-2323, 2009.

[21] N. I. o. A. a. I. Disease. (2015). NIAID Category A, B, and C Priority Pathogens. Available: https://www.niaid.nih.gov/topics/biodefenserelated/biodefense/pages/cata.aspx

[22] D. Benson, M. Cavanaugh, K. Clark, I. Karsch-Mizrachi, D. Lipman, J. Ostell, et al., "GenBank," Nucleic Acids Research, vol. 41, pp. D36-42, 2013.

[23] Y. Chen, C. Lin, C. Wang, H. Wu, and P. Hwant, "An optimized procedure greatly improves EST vector contamination removal," BMC Genomics, vol. 8, 2007.

[24] P. Bork and A. Bairoch, "Go hunting in sequence databases but watch out for the traps," Trends Genet, vol. 12, pp. 425-427, 1996.

[25] "Quality control in databanks for molecular biology," Bioessays, vol. 22, pp. 1024-1034, 2000.

[26] G. Seluja, A. Farmer, M. McLeod, C. Harger, and P. Schad, "Establishing a method of vector contamination identification in database sequenes," Bioinformatics vol. 15, pp. 106-110, 1999.

[27] A. Shcherbina, "FASTQSim: platform-independent data characterization and in silico read generation for NGS datasets," BMC Research Notes, vol. 7, p. 533, 2014.

[28] P. Cock, T. Antao, J. Chang, B. Chapman, C. Cox, A. Dalke, et al., "Biopython: freely available Python tools for computational molecular biology and bioinformatics," Bioinformatics, vol. 25, pp. 1422-1423, 2009.

[29] D. O. Ricke. (2011). GenBankParser. Available: https://github.com/doricke/BioTools/tree/master/GenBankParser

[30] B. Ondov, N. Bergman, and A. Phillippy, "Interactive metagenomic visualization in a Web browser," BMC Bioinformatics, vol. 12, 2011.

[31] S. Naccache, S. Federman, N. Veeraraghavan, M. Zaharia, D. Lee, E. Samayoa, et al., "A cloudcompatible bioinformatics pipeline for ultrarapid pathogen identification from next-generation sequencing of clinical samples," Genome Research, vol. 24, pp. 1180-1192, 2014.

[32] N. Daniels, A. Gallant, J. Peng, L. Cowen, M. Baym, and B. Berger, "Compressive genomics for protein databases," Bioinformatics, vol. 29, pp. i283-i290, 2013.

[33] D. Huang, B. Sherman, and R. Lempicki, "Systematic and integrative analysis of large gene lists using DAVID Bioinformatics Resources," Nature Protocols, vol. 4, pp. 44-57, 2009.

[34] C. Alkan, S. Sajjadian, and E. Eichler, "Limitations of next-generation genome sequence assembly," Nature Methods, vol. 8, pp. 61-65, 2010.

[35] T. Smith and S. Porter, "Development and role of the human reference sequence in personal genomics," Wiley Online Library, 2014. 
[36] N. Sarkonen, "Oral Actinomyces Species in Health and Disease: Identification, Occurence and Importance of Early Colonization," ed: National Public Health Institute, 2007.

[37] R. Vijayakumar, T. Sandle, and C. Manoharan, "Review of fungal contamination in pharmaceutical products and phenotypic identification of contaminants by conventional methods," European Journal of Parenteral and Pharmaceutical Sciences, vol. 17, pp. 4-19, 2012. M. Ndao, "Diagnosis of parasitic diseases: old and new approaches," Interdisciplinary Perspectives on Infectious Diseases, vol. 2009, 2009. 


\section{Figures}

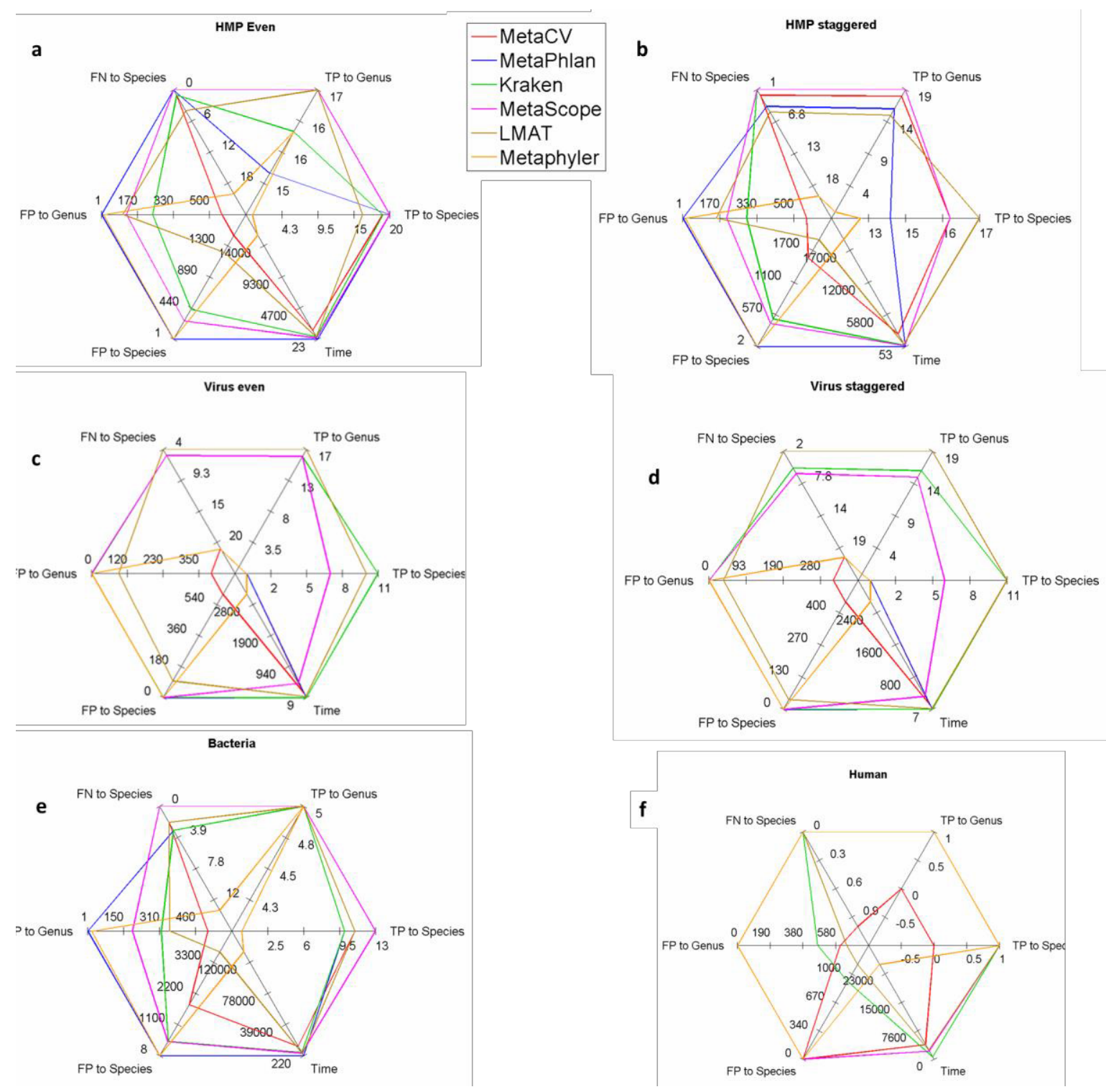

gure 1. Performance metrics for 6 metagenomic analysis algorithms across the 6 in silico evaluation datasets. Algorithms evaluated ıclude MetaCV (red line), MetaPhIAn (blue line), Kraken (green line), MetaScope (pink line), LMAT (brown line), MetaPhyler (orange רe). Metrics evaluated include true positives (TP) to genus level, TP to species level, false positives (FP) to genus level, FP to species :vel, false negatives (FN) to species level, and runtime in seconds. Values indicative of high performance are at the periphery of the idar plot, values indicative of poor performance are at the center of the plot. a. HMP dataset with even coverage. b. HMP dataset 'ith staggered coverage. c. Virus dataset with even coverage. d. Virus dataset with staggered coverage. e. Bacterial dataset. f. Human ataset. The MetaPhIAn algorithm failed to run on the human dataset. 
bioRxiv preprint doi: https://doi.org/10.1101/046532; this version posted March 31, 2016. The copyright holder for this preprint (which was not certified by peer review) is the author/funder, who has granted bioRxiv a license to display the preprint in perpetuity. It is made available under aCC-BY 4.0 International license.

a HMP Even, Genus

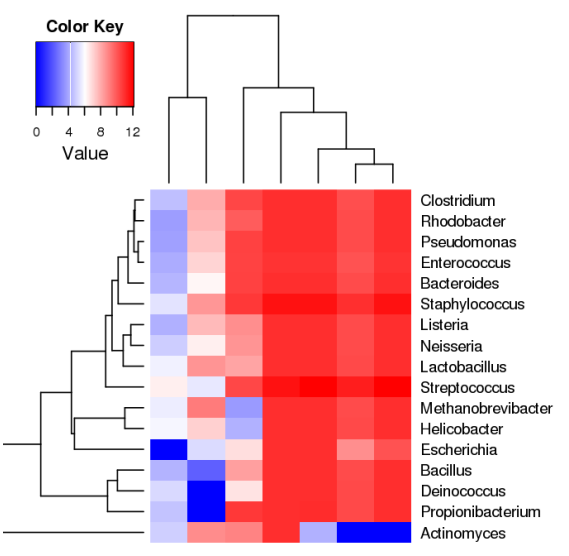

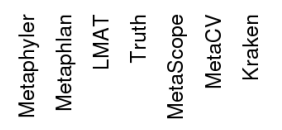

d HMP Even, Species

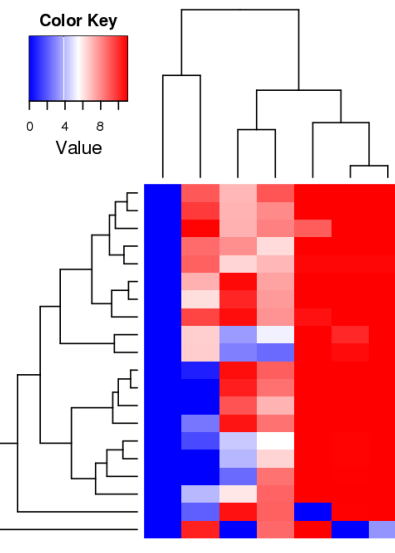

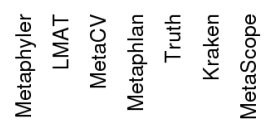
Deinococcus radiodurans
Bacteroides vulgatus Enterocococuus faecalis Escherichia coli Bacillus corrous
Streptocococus mutans Streptocococcus mutans
Lactobacillus gasseri Helicobacter pylori Naisseria maningitidis

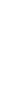

b HMP Staggered, Genus

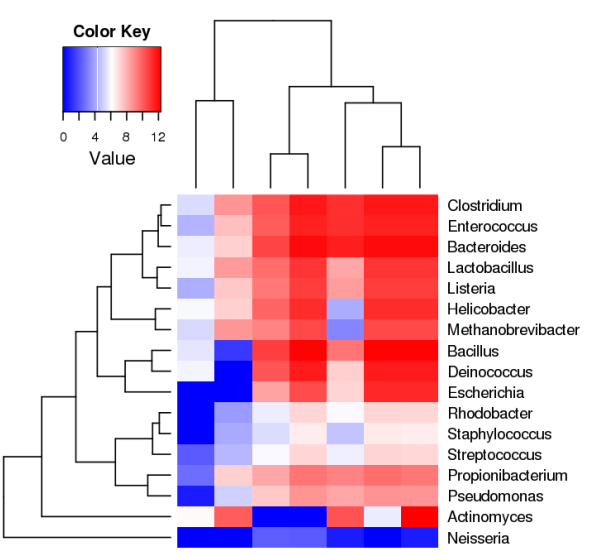

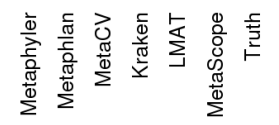

e HMP Staggered, Species
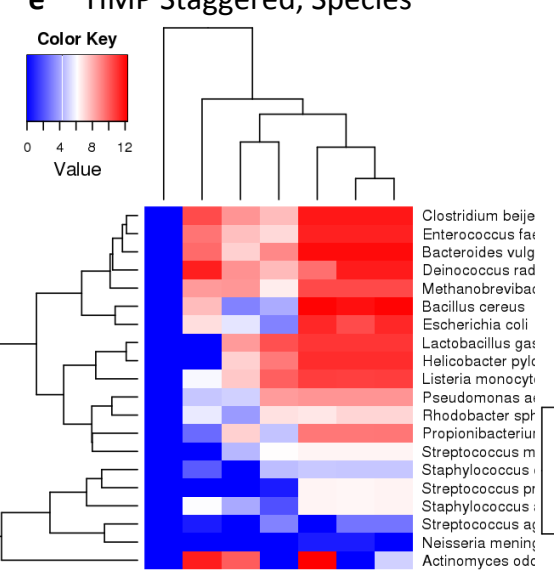

Methanobrevibacter smithi Clostridium beilertinckii Pseudomonas aeruginos
Listeria monocoytogenes Rhodobacter sphaeroides Staphylococcus epidermidis Streptococcus pneumonia propionibacterium acnes Strepto coccus agalactiae
Actinomyces odontolyticus c Bacteria, Genus

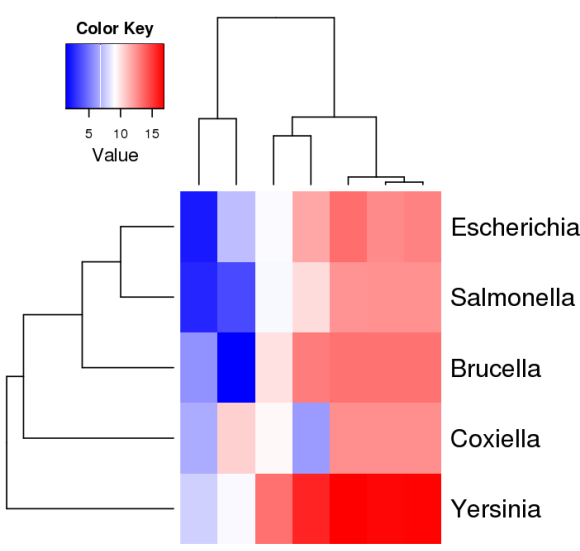

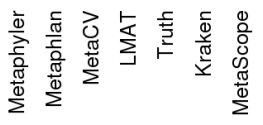

f Bacteria, Species

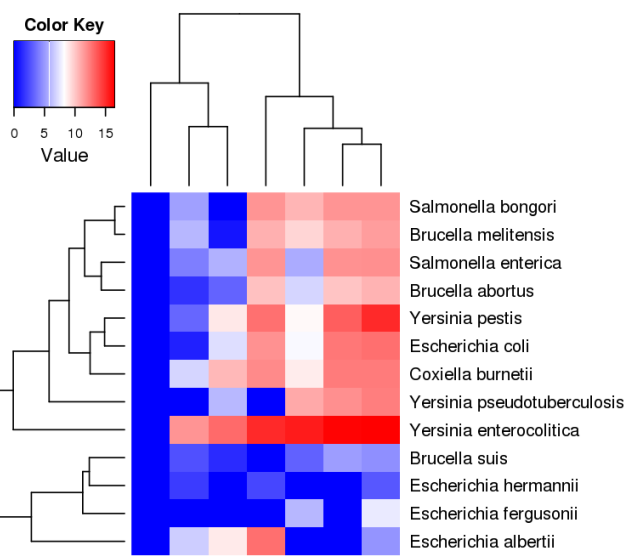

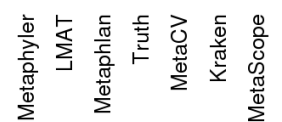



aCC-BY 4.0 International license.

g Virus Even, Genus
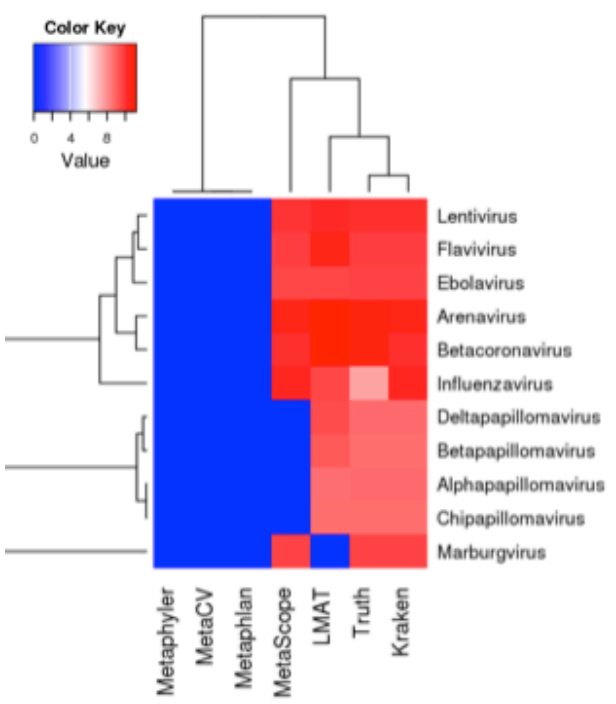

i Virus Even, Species
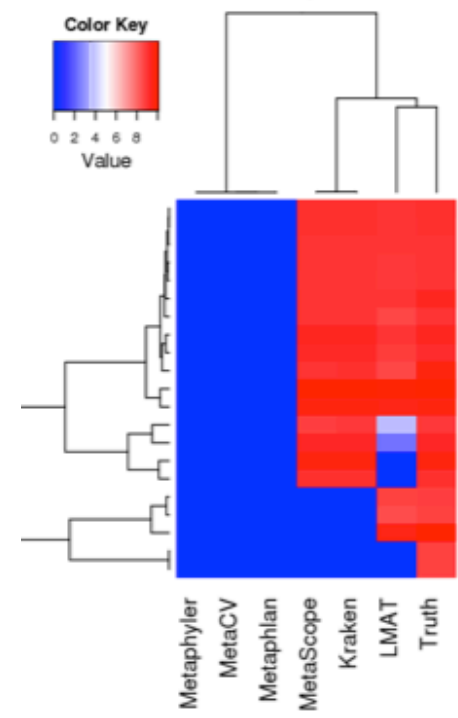

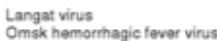
Omsk hamorrthag Sabia virus
Guanarito virus
SiV Chaparo virus HIV 2
Influenza B virus
Intluanza C vinus Influanza C visus Human corenavirus HKU, Sudan ebelavirus
HIV 1 Influenza A virus Marburg virus European aik papillomavirus Human papillomavirus 5 SARS coronavirus Hurnan papillomavirus 32 h Virus Staggered, Genus
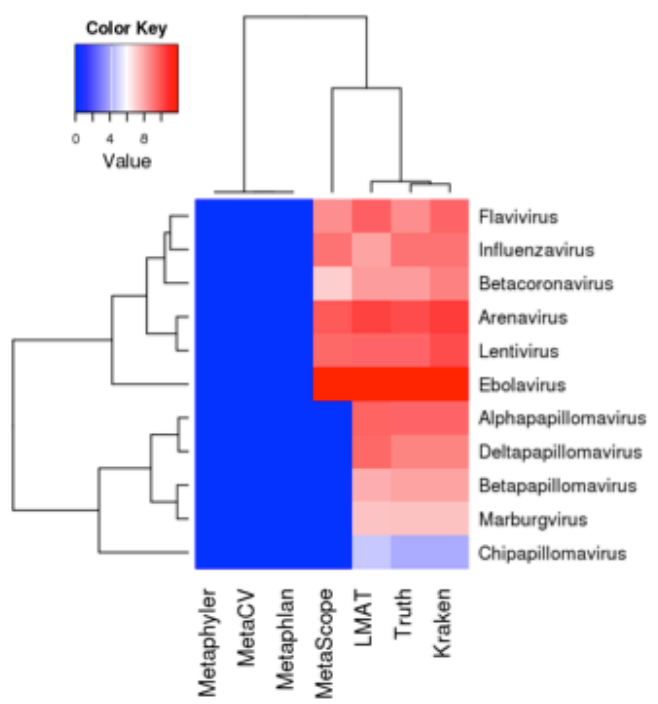

j Virus Staggered, Species

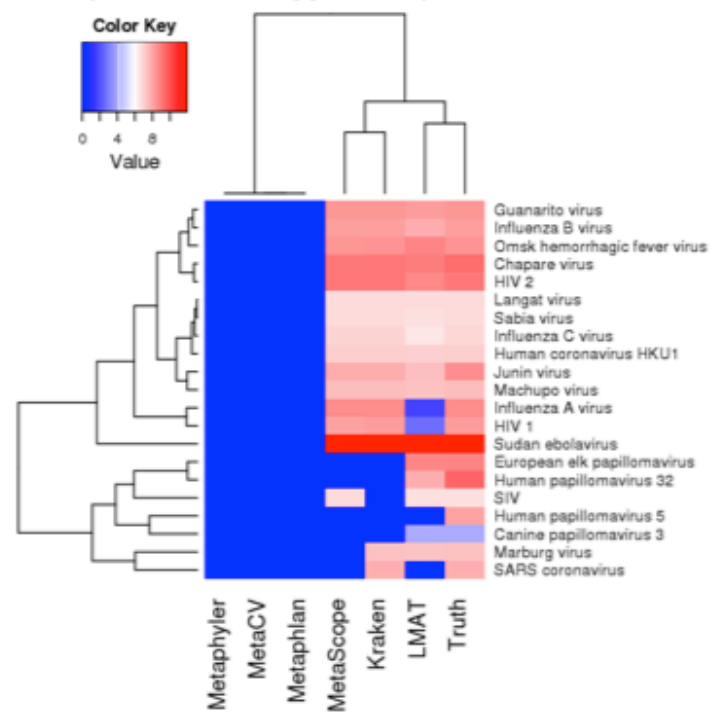

k Human host

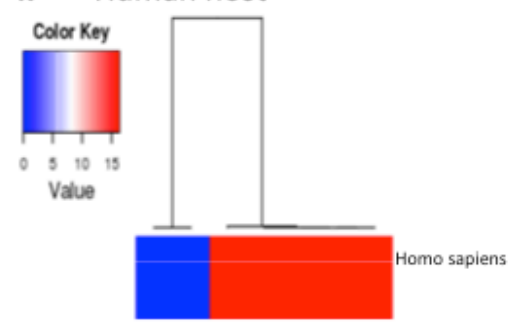

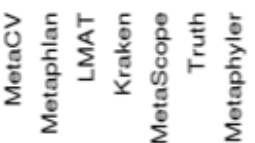

Figure 2. Number of correctly assigned reads to each organism at the genus and species level. Heatmap color scales are log10 (number of correctly assigned reads). The "Truth" column indicates the number of reads spiked into the FASTQ input file for the specified genus or species. a. - e. Reads mapped correctly to the genus level for the HMP even, HMP staggered, bacteria, virus even, virus staggered datasets, respectively. f. - k. Reads mapped correctly to the species level for HMP even, HMP staggered, bacteria, virus even, virus staggered, and human datasets, respectively. 
bioRxiv preprint doi: https://doi.org/10.1101/046532; this version posted March 31, 2016. The copyright holder for this preprint (which was not certified by peer review) is the author/funder, who has granted bioRxiv a license to display the preprint in perpetuity. It is made available under aCC-BY 4.0 International license.
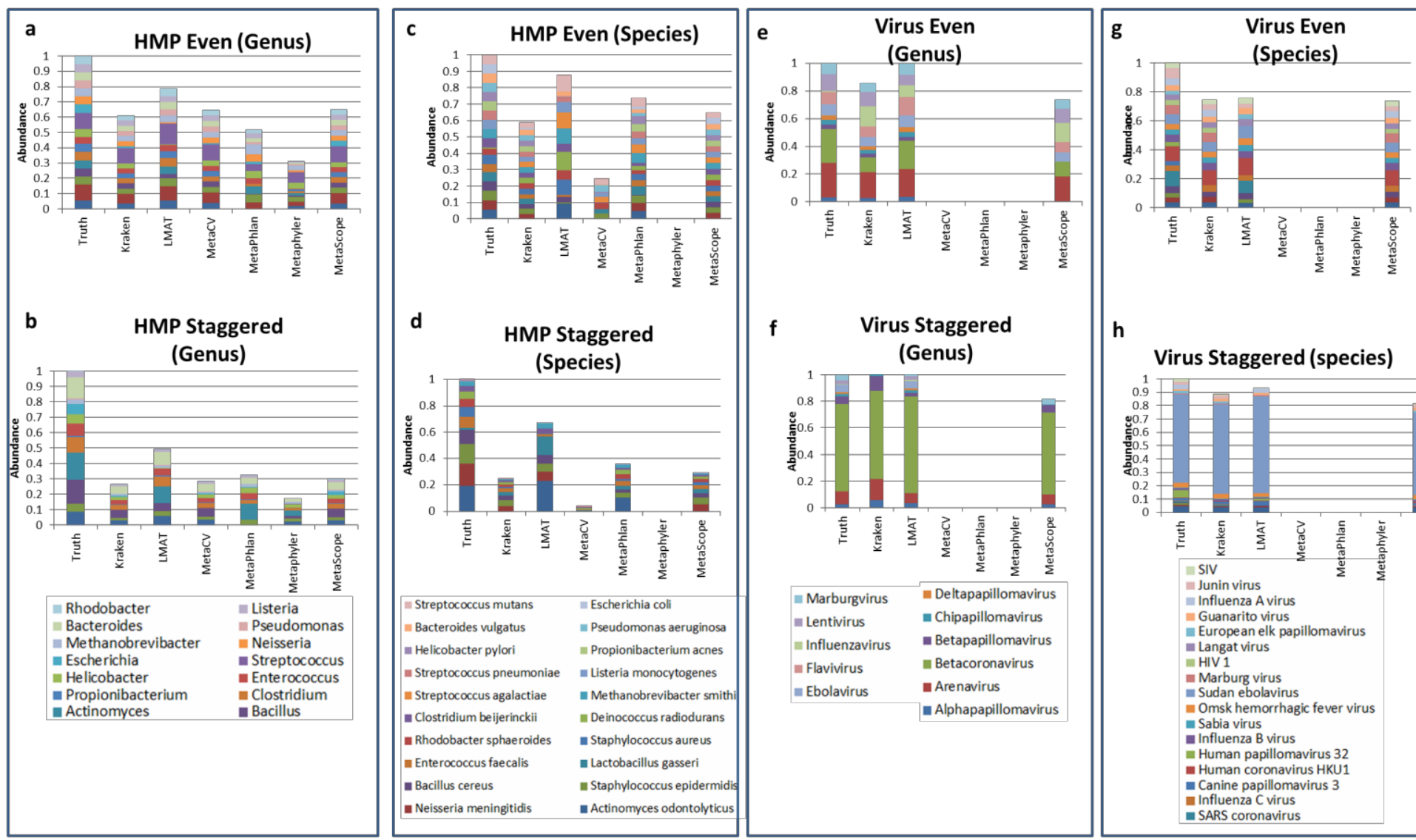

h
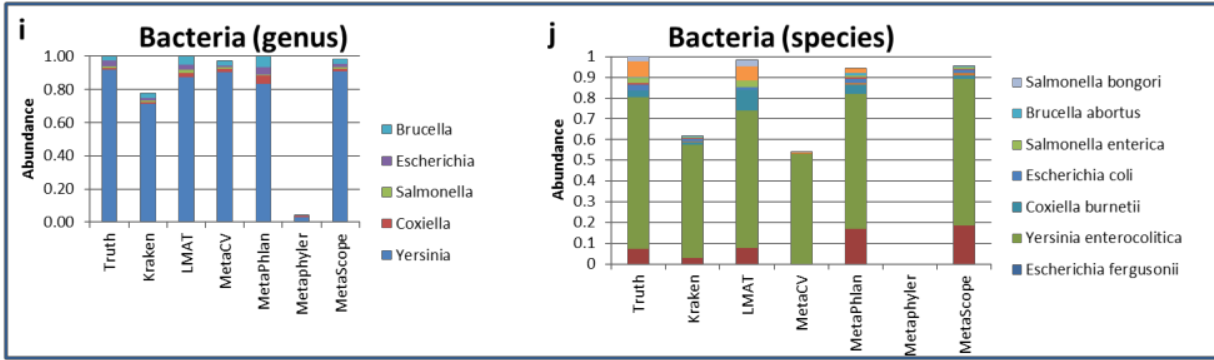

Escherichia albertii

- Escherichia hermanni

- Brucella melitensis

- Yersinia pseudotuberculosis

- Brucella suis

- Yersinia pestis

Figure 3. Relative abundance of organisms to the species and genus level. "Truth" column indicates relative abundance of genera and species added to the in silico FASTQ input file. 
a Bacteria (genus) b Bacteria (species) c Bacteria (genus) Top hits d Bacteria (species) Top hits

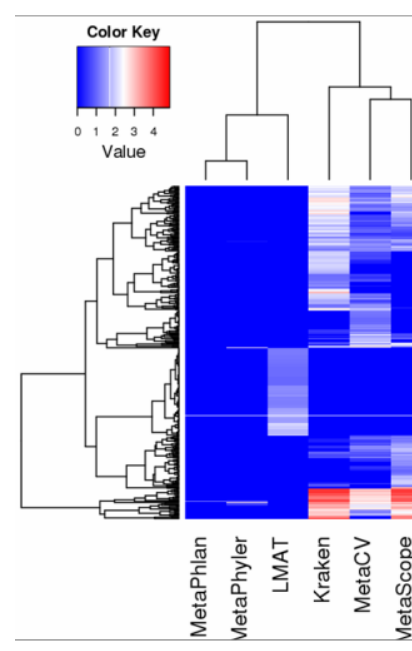

Virus even (genus)

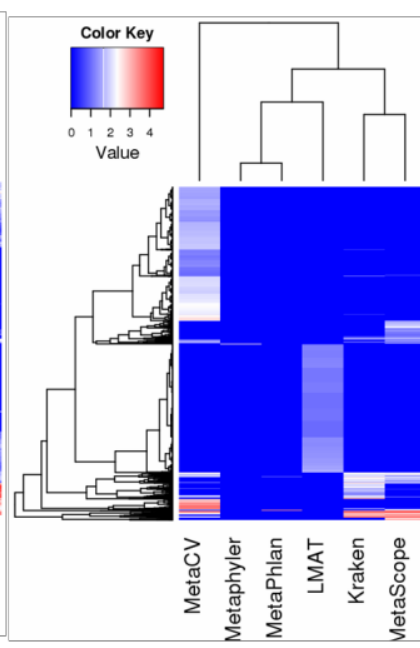

Virus even (species)

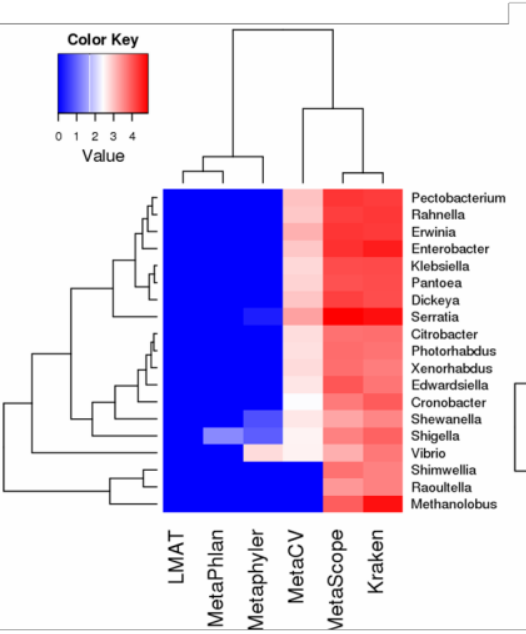

Virus even (genus)

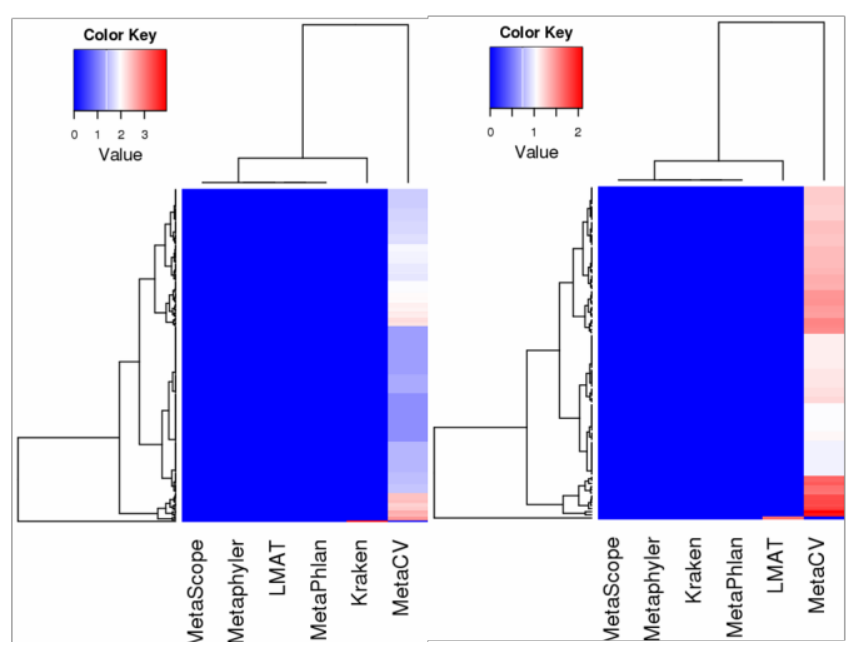

g Top hits

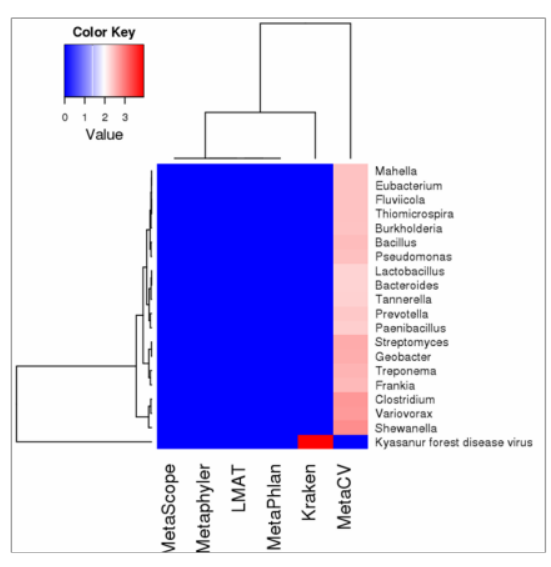

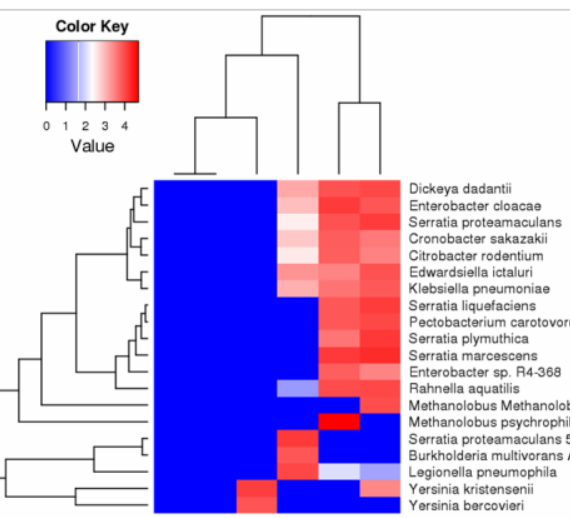

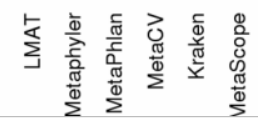

Virus even (species)

h Top hits

Figure 4. False positive organisms identified to the genus and species level by the 6 metagenomic algorithms. Heatmap color scales are $\log 10$ (number of incorrectly assigned false positive reads) for a genus or species. a. All false positive genera identified in the bacterial dataset. b. All false positive species identified in the bacterial dataset. c. 20 false positive genera for the bacterial dataset with the most assigned reads. $\mathbf{d}$. 20 false positive species for the bacterial dataset with the most assigned reads. e. All false positive genera identified in the virus even dataset. $f$. All false positive species identified in the virus even dataset. g. 20 false positive genera for the virus even dataset with the most assigned reads.

h. 20 false positive species for the virus even dataset with the most assigned reads. 

aCC-BY 4.0 International license.
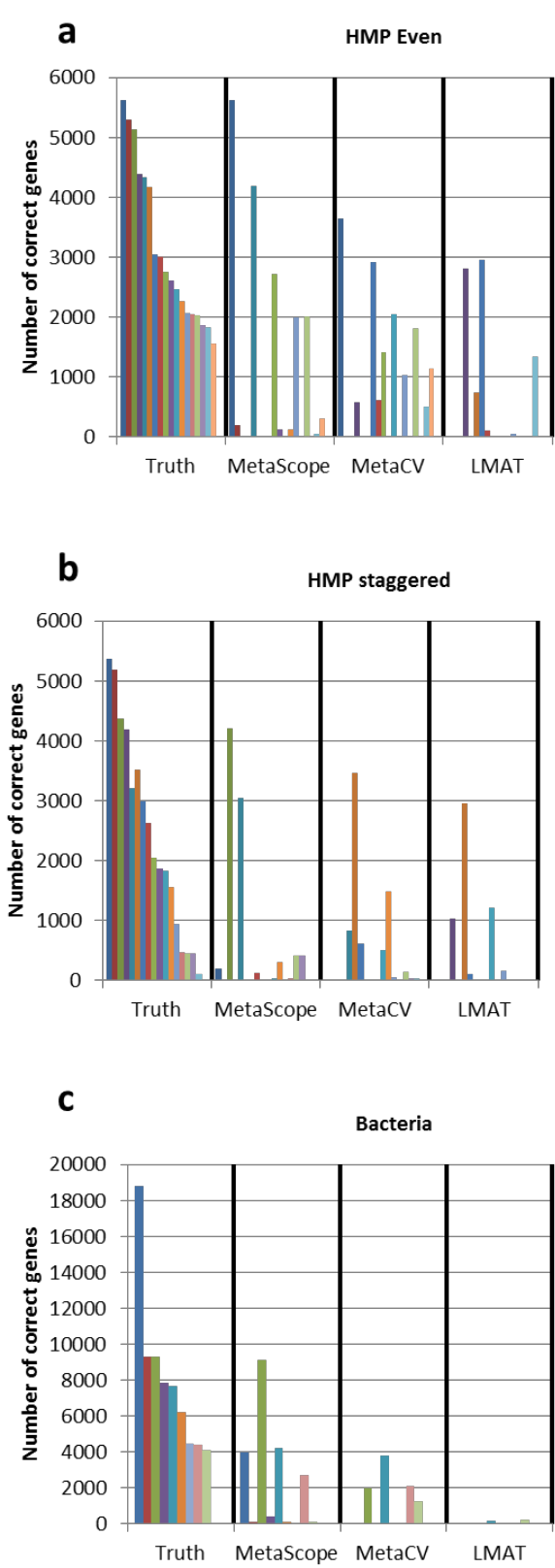

- Pseudomonas aeruginosa - Bacillus cereus = Clostridium beijer inckii - Rhodobacter sphaeroide Escherichia coli - Bacteroides vulgatus Deinococcus radiodurans - Listeria monocytogenes $n$ Staphylococcus aureus. - Enterococcus faecalis Wtaphylococcus epidermidis - $=$ Streptococcus pneumoniae - Actinomyces odontolyticus mStreptococcus mutan - Lactobacillus gasseri Methanobrevibacter smith घelicobacter pylori - $\mathrm{Streptococcus} \mathrm{agalactiae}$ - Neisseria meningitidis

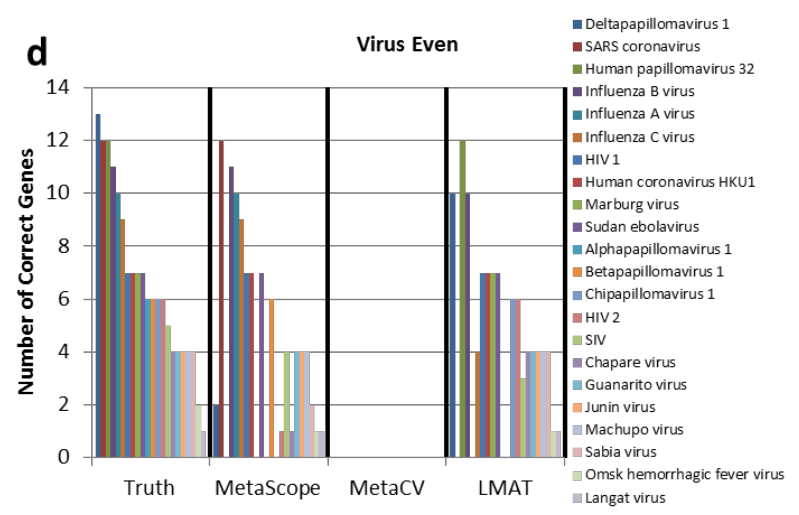

- Bacillus cereus - Clostridium beijer inckii - Escherichia coli - Bacteroides vulgatus - Pseudomonas aeruginosa - Deinococcus radioduran - Dinococcus radiodurans - Enterococcus faecalis - Actinomyces odontolyticus - Lactobacillus gasseri - Methanobrevibacter smithi - Helicobacter pylori - Rhodobacter sphaeroides In Streptococcus pneumoniae Streptococcus mutans - Staphylococcus aureus In Staphylococcus epidermidis - Neisseria meningitidis

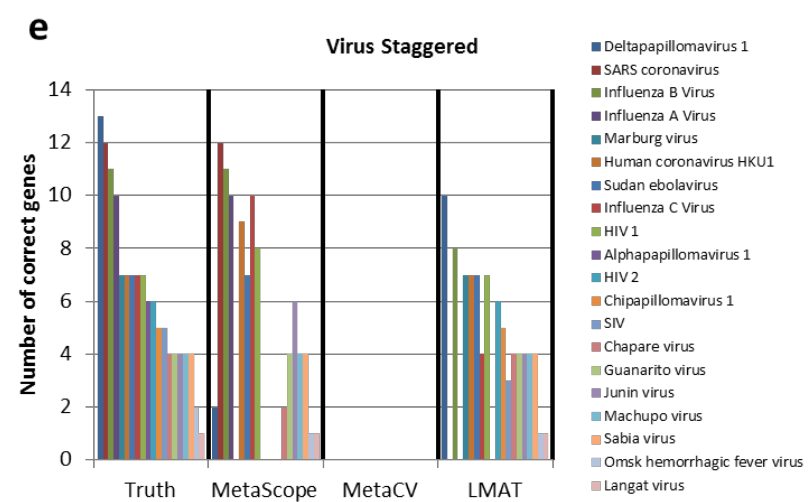

Figure 5. Number of genes correctly identified to the species level across the 5 evaluation datasets (the $6^{\text {th }}$ evaluation dataset consisting of human host reads is not shown). "Truth" column indicates the number of genes with non-zero read coverage in the dataset. MetaScope, MetaCV, and LMAT algorithms provide gene assignment capabilities; Kraken, MetaPhyler, and MetaPhlAn do not call genes and were not included in this evaluation. 


\section{Tables}

Table 1. Radar plot area in normalized units across six evaluation datasets. Higher areas, indicative of better performance, are colored in blue.

\begin{tabular}{|c|c|c|c|c|c|c|c|}
\hline Dataset & Human & Virus Staggered & Virus Even & Bacteria & HMP Staggered & HMP Even & Area Sum \\
\hline MetaScope & 2.54 & 1.90 & 2.14 & 2.23 & 2.03 & 2.32 & 13.15 \\
\hline Kraken & 1.64 & 2.36 & 1.21 & 1.75 & 1.90 & 1.76 & 10.62 \\
\hline LMAT & 1.41 & 2.45 & 2.25 & 1.38 & 1.46 & 1.59 & 10.54 \\
\hline MetaPhIAn & & 0.48 & 0.99 & 2.25 & 1.88 & 2.02 & 7.62 \\
\hline MetaCV & 0.82 & 0.09 & 0.14 & 1.43 & 1.25 & 1.08 & 4.80 \\
\hline MetaPhyler & 1.88 & 0.60 & 0.09 & 0.67 & 0.57 & 0.63 & 4.44 \\
\hline
\end{tabular}

Table 2. Algorithm runtime in seconds across six evaluation datasets.

\begin{tabular}{lcccccc}
\hline \multicolumn{1}{c}{ Dataset } & Human & $\begin{array}{c}\text { Virus } \\
\text { Staggered }\end{array}$ & $\begin{array}{c}\text { Virus } \\
\text { Even }\end{array}$ & Bacteria & $\begin{array}{c}\text { HMP } \\
\text { Staggered }\end{array}$ & HMP Even \\
\hline MetaScope & 2160 & 327 & 427 & 3686 & 261 & 233 \\
Kraken & 600 & 7 & 9 & 4400 & 300 & 400 \\
LMAT & 2428 & 20 & 39 & 2700 & 502 & 427 \\
MetaPhIAn & Seg Fault & 12 & 12 & 220 & 53 & 23 \\
MetaCV & 3873 & 120 & 150 & 11966 & 2337 & 1322 \\
MetaPhyler & 25200 & 2640 & 3100 & 129600 & 19231 & 15480 \\
\hline
\end{tabular}

\section{Supplementary Materials}

Supplementary Table 1. Source organisms and coverage levels for HMP Even and HMP Staggered datasets.

Supplementary Table 2. Source organisms and coverage levels for Bacterial dataset.

Supplementary Table 3. Source organisms and coverage levels for Virus Even and Virus Staggered datasets.

Figure S1. FASTQSim in silico dataset composition to strain level. a. 20 bacteria from the Human Microbiome Project (HMP), even coverage levels. b. Same 20 bacteria from HMP, staggered coverage levels. c. 22 species of viruses across 11 genera, even coverage levels. d. Same 22 species of viruses, staggered coverage levels. e. 33 strains of bacteria representing 13 species and 5 genera. See Krona HTML files for a-e. 
a

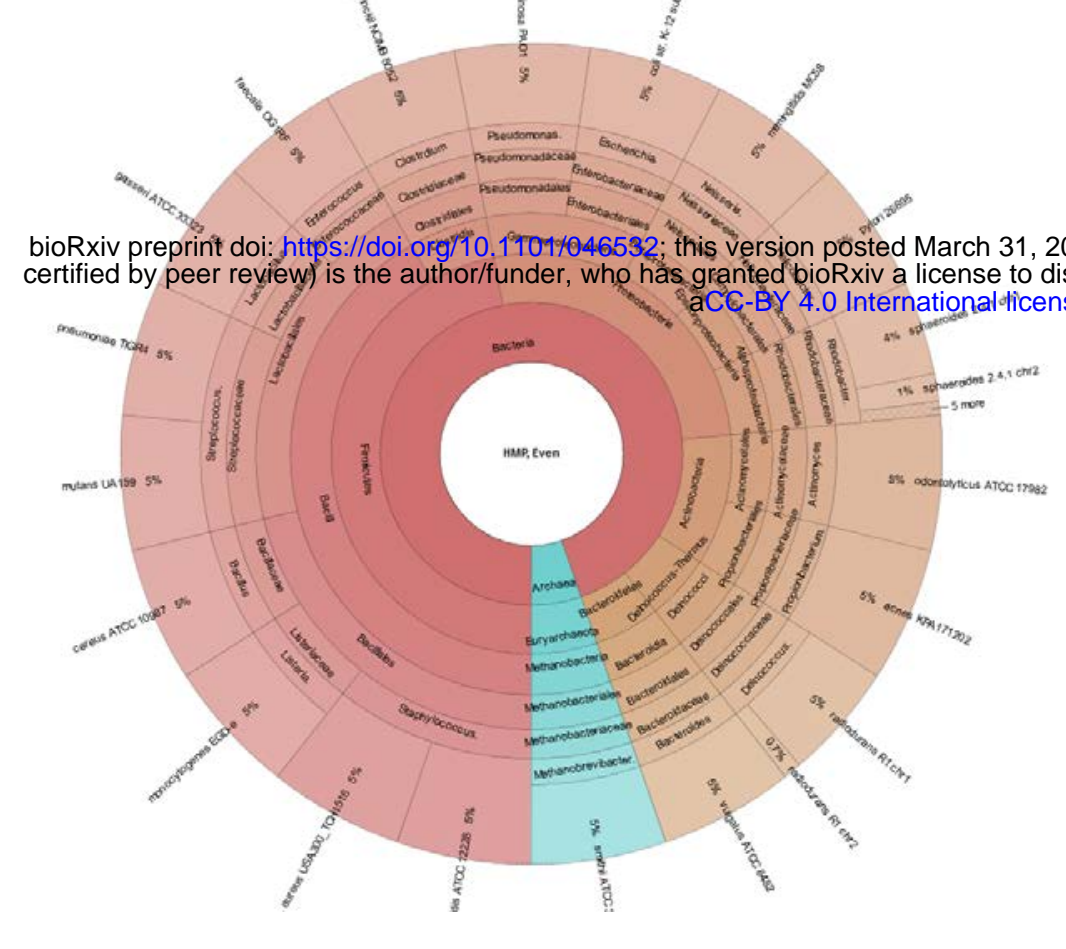

C
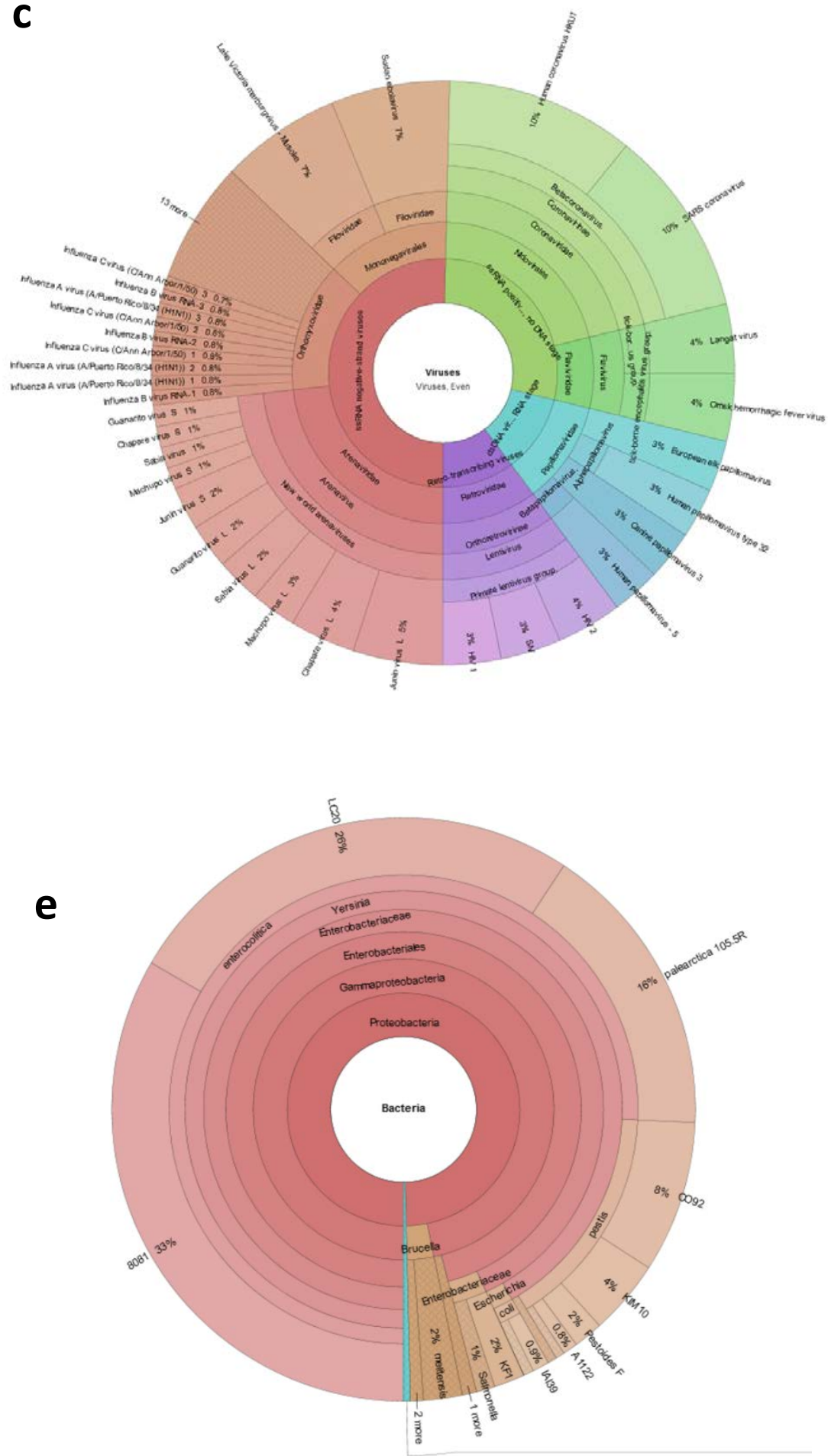
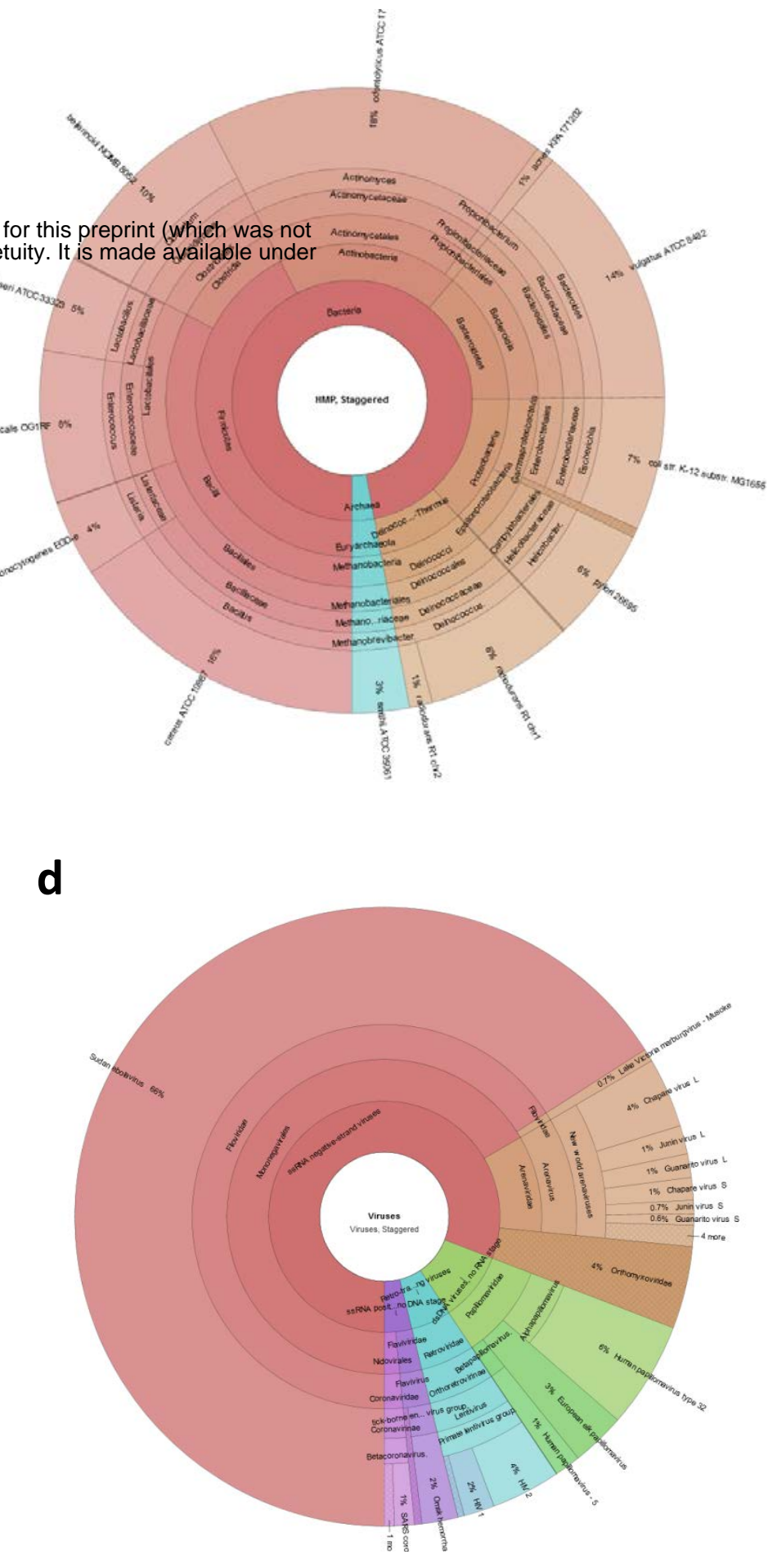